ESAIM: PROCEEDINGS, December 2009, Vol. 29, p. 8-27

F. Coquel, Y. Maday, S. Müller, M. Postel and Q. H. Tran, Editors

\title{
COST-EFFECTIVE MULTIRESOLUTION SCHEMES FOR SHOCK COMPUTATIONS *
}

\author{
Guillaume Chiavassa $^{1}$, Rosa Donat ${ }^{2}$ and Anna Martinez-Gavara ${ }^{3}$
}

\begin{abstract}
Harten's Multiresolution framework has provided a fruitful environment for the development of adaptive codes for hyperbolic PDEs. The so-called cost-effective alternative [4, 8, 21] seeks to achieve savings in the computational cost of the underlying numerical technique, but not in the overall memory requirements of the code. Since the data structure of the basic algorithm does not need to be modified, it provides a set of tools that can be easily implemented into existing codes and that can be very useful in order to speed up the numerical simulations involved in the testing process that is associated to the development of new numerical schemes.

In this paper we present two different applications of the cost-effective multilevel technique developed in [8] in non-standard situations. In both cases, its use has led to a drastic reduction in the computational time required for $2 \mathrm{D}$ numerical simulations, and hence to the ability to perform fine mesh simulation and, hence, to investigate new numerical techniques on personal computers.
\end{abstract}

\section{INTRODUCTION}

The numerical simulation of physical problems modeled by systems of conservation laws is rather delicate, due to the presence of discontinuities in the solution. Modern High-Resolution Shock Capturing (HRSC) schemes succeed in computing highly accurate numerical solutions, typically second, third or even higher order in smooth regions, while maintaining sharp, oscillation-free numerical profiles at discontinuities. HRSC schemes of the ENO-WENO family are well known nowadays in the hyperbolic community, due to their robust behavior in many different scenarios, even though no analytical proof of their convergence is available. There is a large body of scientific activity around these schemes, whose power lies in a sophisticated design of the numerical flux function in a conservative discrete formulation of the hyperbolic PDE or system. This is, in fact, the main drawback of these schemes, especially in multi-dimensional computations involving systems of PDEs, since the numerical flux computations tend to be fairly expensive.

It is well known, however, that the costly numerical flux function of a HRSC scheme is only necessary in a neighborhood of singularities, hence a substantial amount of computational expense could be avoided by reducing the number of evaluations of costly numerical flux functions to those regions where singularities exist or are starting to develop. In the late eighties and early nineties (see e.g. [21]), Harten proposed various strategies to decide the regions in which a HRSC scheme must necessarily be used in order to maintain the high resolution properties in the numerical solution. Harten's original multilevel strategies were developed for finite volume schemes for homogeneous conservation laws [4,21], where the numerical data are naturally treated

* Research supported in part by MTM2008-00974

1 Ecole Centrale Marseille and M2P2, UMR6181

2 Dept. Matematica Aplicada, Universitat de Valencia

3 Dept Matematica Aplicada I. Universidad de Sevilla

(C) EDP Sciences, SMAI 2009 
as approximations to the cell-averages of the true solution. Later on, Chiavassa and Donat [8] applied the cost-reduction strategy to Shu-Osher ENO-type finite-difference schemes, where the data are interpreted as point-values. This allows, in a rather natural way, to think of the basic ingredients of the smoothness analysis as interpolation errors, so that its relation to the smoothness of the underlying function is easy and well understood.

The multilevel technique developed in [8], combined with a HRSC scheme developed in [13], has been applied in a number of situations with success. As observed in $[8,9,32]$, the computational gain can reach a factor of 7 , but normally its range lies between 3 and 5 . In most situations, this is sufficient to obtain high resolution simulations on rather fine grids in $2 \mathrm{D}$. The ability to obtain fine-mesh simulations in $2 \mathrm{D}$ is very relevant, since on many occasions diminishing the size of the computational mesh, and hence the associated numerical viscosity inherent to the scheme, can lead to numerical pathologies which are very hard to observe otherwise.

In this work, we apply a straightforward extension of the multilevel strategy developed in [8] to nonhomogeneous systems of conservation laws. In particular, we shall investigate the properties of the extended multilevel technique on the shallow water system, and on a penalization model for compressible fluid flow with obstacles.

We also mention here that there has been some recent work on the inclusion of source terms in fully adaptive multilevel strategies [24]. Fully adaptive schemes are not only cost-efficient, but also significant memory gains can be achieved through the full exploitation of the multilevel structure of the scheme, see [10] for a detailed account of this technique in the homogeneous case, and [33], [12] for later developments on these fully adaptive multilevel schemes. These techniques do require special data structures in order to obtain the expected memory gains, and its incorporation into an existing code is not straightforward. The cost effective alternative proposed in this work, can be incorporated almost as an external routine, at each time step, and remains the easiest multilevel scheme to adapt to an existing code.

The rest of the paper is organized as follows: The basic cost-effective multilevel algorithm is briefly recalled in section 1 . In section 2 , we describe the shallow water system and the two numerical schemes we consider for its numerical approximation. In section 3 we present various numerical experiments that demonstrate that our multilevel strategy respects the well balancing properties of the underlying HRSC scheme, which is a fundamental property in shallow water simulations. In section 4 we recall a penalization technique for compressible flow with obstacles, developed in [5] and show several novel simulations that also illustrate the versatility of the numerical technique in obtaining fine-mesh simulations in non-standard situations.

\section{The multilevel Algorithm}

The general setting of the multilevel strategy we shall employ here has been described and analyzed in [8] for homogeneous conservation laws. In what follows we recall the main steps and explain our extension to the non-homogeneous case.

Let us consider a 2D system of hyperbolic conservation laws

$$
\partial_{t} \vec{U}+\vec{\nabla} \cdot \overrightarrow{\mathcal{F}}(\vec{U})=\mathcal{B}
$$

where $\vec{U}$ is the vector of conserved quantities, and $\mathcal{B}=\mathcal{B}(\vec{U}, \vec{\nabla} \vec{U})$ represents the contribution of all terms not included in the divergence term. We shall only consider discretizations of this system on a Cartesian grid $\chi^{0}=\left\{\left(x_{i}=i \triangle x, y_{j}=j \triangle y\right), \quad i=0, \cdots, N_{x} \quad j=0, \cdots, N_{y}\right\}$ using the semi-discrete formulation

$$
\partial_{t} \vec{U}_{i j}+\mathcal{D}_{i j}=\mathcal{B}_{i j}
$$

It is assumed that the numerical divergence is computed as

$$
\mathcal{D}_{i j}=\frac{\vec{F}_{i+\frac{1}{2}, j}-\vec{F}_{i-\frac{1}{2}, j}}{\triangle x}+\frac{\vec{G}_{i, j+\frac{1}{2}}-\vec{G}_{i, j-\frac{1}{2}}}{\triangle y},
$$


where $\vec{F}$ and $\vec{G}$ are consistent numerical (normal) flux functions, which define the HRSC scheme used on a given application. The goal of the multilevel method is to reduce the CPU time associated to the underlying scheme by reducing the number of expensive flux evaluations involved in the computation of the numerical divergence. The computation of $\mathcal{B}_{i j}$ depends on the problem at hand, and we shall present several situations in the following sections.

By rewriting (2) as

$$
\partial_{t} \vec{U}_{i j}+\left(\mathcal{D}_{i j}-\mathcal{B}_{i j}\right)=0
$$

the basic mechanism of the cost-effective alternative for these problems is essentially that considered in [8], since the smoothness analysis of the solution vector $\vec{U}_{i j}$ reveals the behavior of the extended divergence $\mathcal{G}_{i j}=\mathcal{D}_{i j}-\mathcal{B}_{i j}$ with respect to smoothness.

\subsection{Smoothness analysis by Interpolatory Wavelet Transforms}

The use of an interpolatory wavelet transform in this context, as proposed in [8], is justified by the fact that in the finite-difference framework for ENO schemes proposed by Shu and Osher in [35], $\vec{U}_{i j} \approx u\left(x_{i}, y_{j}\right)$. The Shu-Osher framework is used in all the numerical techniques we propose in this paper.

In what follows, we shall restrict ourselves to $1 \mathrm{D}$ for simplicity in the description. The $2 \mathrm{D}$ extension is carried out by a standard tensor product construction. The different resolution levels are specified by a set of nested grids $\left\{\chi^{l}, \quad l=0,1, \ldots, L\right\}$, where $\chi^{0}$ is considered the finest grid. The nested structure in the grid hierarchy implies $x_{i} \in \chi^{l} \Longleftrightarrow x_{2^{l} i} \in \chi^{0}$. If $\left(v_{i}^{0}\right)_{i}$ are the point-values of a function $v$ on $\chi^{0}$, due to the embedding of the grids we have

$$
v_{i}^{l}=v_{2^{l} i}^{0}, \quad i=0, \ldots, N_{l}=N_{x} / 2^{l}, \quad v_{i}^{l}=v_{2 i}^{l-1} \quad i \in N_{l} .
$$

From the discrete data on $\chi^{l}$ a set of predicted values on $\chi^{l-1}$ (the next finer level on the resolution ladder) can easily be computed by polynomial interpolation. Hence a set of predicted values, $\tilde{v}_{i}^{l-1}$, can be computed by considering a centered interpolatory technique, in order to achieve maximal accuracy,

$$
\begin{aligned}
& \tilde{v}_{i}^{l-1}=v_{i / 2}^{l} \quad \text { if } x_{i} \in \chi^{l} \\
& \tilde{v}_{i}^{l-1}=\mathcal{I}\left[x_{i} ; v^{l}\right]=\sum_{k=1}^{s} \beta_{k}\left(v_{i+k-1}^{l}+v_{i-k}^{l}\right) \quad \text { if } x_{i} \in \chi^{l-1} \backslash \chi^{l} .
\end{aligned}
$$

The coefficients $\beta_{k}, k=1, \ldots, s$ can be easily determined (see e.g. [4]).

The wavelet coefficients represent the difference between exact and predicted data, $d_{i}^{l}=v_{i}^{l-1}-\tilde{v}_{i}^{l-1}, \quad x_{i} \in$ $\chi^{l-1}$ and, in this framework, they are interpolation errors which can be used directly as "regularity sensors" in order to localize non-smooth behavior. Notice that (6) implies that $d_{i}^{l}=0$ for $i \in \chi^{l}$, hence we can write

$$
\begin{aligned}
v_{i}^{l-1}=v_{i / 2}^{l}, & & \text { if } x_{i} \in \chi^{l} \\
v_{i}^{l-1}=\tilde{v}_{i}^{l-1}+d_{i}^{l} & & \text { if } x_{i} \in \chi^{l-1} \backslash \chi^{l} .
\end{aligned}
$$

The sets $\left\{v^{l}\right\}$ and $\left\{v^{l-1}, d^{l}\right\}$ are algebraically equivalent, and the same can be said about the sets

$$
v^{0} \leftrightarrow \quad M v^{0}=\left(v^{L}, d^{1}, \ldots, d^{0}\right) .
$$

Let us consider a Forward-Euler time-discretization of a 1D version of (4), i.e.,

$$
U_{i}^{n+1}=U_{i}^{n}-\triangle t \mathcal{G}_{i}^{n} .
$$

The application of any linear multiscale transformation $M$ to this relation leads to

$$
M U_{i}^{n+1}-M U_{i}^{n}=\triangle t M \mathcal{G}_{i}^{n} .
$$


Hence, it is reasonable to assume that if a spatial location has associated small detail coefficients in both $M U^{n}$ and $M U^{n+1}$, it would also have associated a small detail coefficient in $M \mathcal{G}^{n}$. Hence, at this location, the value of $\mathcal{G}_{i}^{n}$ might be correctly recovered simply by interpolating from lower resolution data. By using this heuristic argument, the information about the regularity of the data contained in $M U^{n}$ is used to determine a flag vector $\left(b_{i}^{l}\right)_{l, i}$, whose value is either 0 or 1 for each spatial location according to the following rule:

$$
\begin{aligned}
& \text { if }\left|d_{i}^{l}\right| \geq \varepsilon \Rightarrow b_{i-k}^{l}=1 \quad k, m=-2, \ldots, 2 \\
& \text { if }\left|d_{i}^{l}\right| \geq 2^{r} \varepsilon \text { and } l>0 \Rightarrow b_{2 i-k}^{l-1}=1 \quad k, m=-1,0,1
\end{aligned}
$$

where $r=2 s$ is the accuracy of the polynomial interpolatory technique used in the prediction step.

The determination of the flag vector above follows Harten's heuristics [21]. On one hand, it takes into account that large values of the detail coefficients correspond to non-smooth zones of the solution, like well resolved shocks or contact discontinuities. In addition, compression regions leading to shock formation, or steep profiles corresponding to discontinuities in the solution, exhibit a lack of regularity that can be estimated by looking at the decay rate of the detail coefficients across scales. Finally, a safety region of two cells around each flagged position is added. This produces a flagged region that should contain the singularities of both $U^{n}$ and $U^{n+1}$ (unknown at time $n$ ), since discontinuities of hyperbolic conservation laws move through the computational domain with a finite speed of propagation, and the CFL stability condition ensures that existing singularities do not travel farther than one cell per time step.

\subsection{The multilevel evaluation of the discrete divergence}

The key point in the design of the multilevel algorithm in [8] consists in substituting the direct computation of the numerical divergence $\mathcal{D}$ on the finest grid by a multilevel strategy. The extended strategy we propose for the non-homogeneous case proceeds in the same manner, but instead of the values of the divergence operator, we apply the multilevel strategy proposed in [8] to the computation of the discrete $\mathcal{G}$ values. Notice that by using the smoothness analysis of $U^{n}$ to compute the flag vector that determines the multilevel computation of $\mathcal{G}$, we implicitly assume that the requirements of Harten's heuristics remain unchanged: finite speed of propagation of singularities, controlled by the CFL condition for stability in the numerical scheme.

The multilevel computation of $\mathcal{G}^{n}$ is, then, carried out as follows: Assuming that $\mathcal{G}^{n}$ is known on $\chi^{l}$, the values of $\mathcal{G}^{l-1}$ on $\chi^{l-1}$ are computed as specified below

- If $x_{i} \in \chi^{l}$, then $\mathcal{G}_{i}^{l-1}=\mathcal{G}_{i / 2}^{l}$.

- If $x_{i} \in \chi^{l-1} \backslash \chi^{l}$, then $\mathcal{G}_{i}^{l-1}$ is computed using the boolean flag as follows:

$$
\begin{aligned}
& \text { if } b_{i}^{l}=1, \quad \text { compute } \mathcal{G}_{i}^{l-1} \text { directly with the HRSC scheme } \\
& \text { if } b_{i}^{l}=0, \quad \text { compute } \mathcal{D}_{i}^{l-1}=\mathcal{I}\left[x_{i} ; \mathcal{G}^{l}\right]=\sum_{k=1}^{s} \beta_{k}\left(\mathcal{G}_{i+k-1}^{l}+\mathcal{G}_{i-k}^{l}\right) .
\end{aligned}
$$

The process is repeated from $l=L, \ldots, 1$ and, once it is completed, we obtain the values of $\mathcal{G}^{n}$ on the finest grid $\chi^{0}$, which are needed by the ODE solver.

\section{The SHALlOW WATER EQUATIONS}

The shallow water equations form a hyperbolic system of conservation laws that approximately describes various geophysical flows. When the effect of the bathymetry, or bottom surface elevation, is taken into account, the equations take the following form

$$
\partial_{t} \vec{U}+\partial_{x} \vec{F}(\vec{U})+\partial_{y} \vec{G}(\vec{U})=S(\vec{x}, \vec{U})
$$


where $\vec{U}=\left(h, q_{1}, q_{2}\right)$ is the vector of unknowns, with $h$ being the water depth and $q_{1}=h u, q_{2}=h v$ the two components of the discharge $((u, v)$ is the velocity vector). The bottom topography is specified by the function $z(x)$. The explicit expression of the $2 \mathrm{D}$ system is

$$
\partial_{t}\left(\begin{array}{c}
h \\
q_{1} \\
q_{2}
\end{array}\right)+\partial_{x}\left(\begin{array}{c}
q_{1} \\
\frac{q_{1}^{2}}{h}+\frac{1}{2} g h^{2} \\
\frac{q_{1} q_{2}}{h}
\end{array}\right)+\partial_{y}\left(\begin{array}{c}
q_{2} \\
\frac{q_{1} q_{2}}{h} \\
\frac{q_{2}^{2}}{h}+\frac{1}{2} g h^{2}
\end{array}\right)=\left(\begin{array}{c}
0 \\
-g h z_{x} \\
-g h z_{y}
\end{array}\right) .
$$

Non-trivial steady state solutions to (12) are physically relevant in many shallow water applications, and exist due to a balance of the flux divergence and the source term related to the variable bathymetry. Numerically preserving non-trivial steady states, or resolving small perturbations to them, when

$$
\partial_{x} \vec{F}(\vec{U})+\partial_{y} \vec{G}(\vec{U}) \approx S(\vec{x}, \vec{U})
$$

yet both terms are relatively large, is a well known difficulty that has received considerable attention in the literature. In particular, the common fractional splitting approach to solve separately the convection and the source terms is known to produce spurious waves of a purely numerical nature. After many time steps, these disturbances can completely destroy the accuracy of the numerical solution.

Much research has been devoted recently to finding numerical schemes that can accurately preserve non-trivial steady states at the discrete level. These schemes are called Well Balanced (WB) after the work of Greenberg and Le Roux $[19,20]$, or are said to satisfy the exact C-property, after the independent work of Bermudez and Vazquez-Cendon [3].

Well Balanced schemes, or schemes that preserve the C-property, incorporate an upwind treatment of the source term. This can be achieved by an appropriate de-centering of the source term contribution, as in e.g. [3], or by the use of specific Riemann solvers (e.g. [20,25])

In this paper we shall consider two numerical schemes recently described in [6] and [27]. Both schemes incorporate an idea due to Gascon and Corberan [17] that becomes very useful in order to design well balanced schemes for balance laws. The balance law is first rewritten in a pseudo-conservative formulation, i.e.

$$
u_{t}+f(u)_{x}=s(x, u), \quad \equiv \quad u_{t}+g_{x}=0
$$

with

$$
g(x, t)=f(u(x, t))-\int_{\bar{x}}^{x} s(y, u(y, t)) d y .
$$

Here $\bar{x}$ is a reference point which can be taken as the initial point in the computational domain, for example.

Both schemes follow the guidelines established by Shu and Osher in [35], the extension to 2D is done dimension by dimension, and the setting is specifically well adapted to the multilevel strategy described in the previous section. The specific details of both schemes are, however, quite different and, for the sake of completeness, we briefly review their essential design next. We refer the interested reader to [6,27] for a full description of the schemes.

\subsection{The 1J-2J scheme}

In [6] the authors extend the 2-Jacobian scheme developed in [13] for homogeneous systems of conservation laws (see also [14]) to the shallow water system. For high order simulations, the standard technique of separating the issues of spatial and temporal accuracy by a method of lines approach is followed, and the time discretization is carried out by a TVD-Runge Kutta scheme [35]. A brief algorithmic description of the numerical scheme is outlined next. 
For the scalar balance law (13), a method of lines discretization of the form

$$
u_{t}+\frac{\hat{g}_{i+\frac{1}{2}}-\hat{g}_{i-\frac{1}{2}}}{\triangle x}=0
$$

is proposed, and the combined numerical fluxes $\hat{g}_{i \pm \frac{1}{2}}$ evaluated in an ENO fashion, as specified in [35], using $f^{\prime}(u)$ for determining the upwind direction. This procedure would involve integrations over domains of the form $\left[\bar{x}, x_{i}\right]$ in the determination of the first-order terms in the expressions of $\hat{g}_{i \pm \frac{1}{2}}$, hence straightforward manipulations are carried out in order to arrive to an equivalent expression that only involves integration on consecutive cells. The equivalent form can be written as

$$
u_{t}+\frac{g_{i+\frac{1}{2}}^{+}-g_{i-\frac{1}{2}}^{-}}{\triangle x}=0
$$

It is observed in $[6]$ that

$$
g_{i+\frac{1}{2}}^{+}-g_{i+\frac{1}{2}}^{-}=\int_{x_{i}}^{x_{i+1}} s(y, u(y, t)) d y,
$$

hence, the signed fluxes $\hat{g}_{i \pm \frac{1}{2}}^{ \pm}$provide an upwind splitting of the source term contribution at the $i+1 / 2$ interface.

The 1D shallow water system,

$$
U_{t}+F(U)_{x}=S(U, x)
$$

is first rewritten in the form

$$
U_{t}+G(U)_{x}=0
$$

with $G(x, t)=F(U(x, t))+B(x, t)$ and $B(x, t)=\left(0, \int_{\bar{x}}^{x} g h z_{x} d s\right)^{T}$. Then, the semi-discrete formulation of the numerical scheme for this $1 \mathrm{D}$ system can be expressed as

$$
U_{t}+\frac{G_{i+\frac{1}{2}}^{+}-G_{i-\frac{1}{2}}^{-}}{\triangle x}=0
$$

where, again, the computation of $G_{i+\frac{1}{2}}^{ \pm}$only involves integral terms over consecutive cell centers. The construction of $G_{i+\frac{1}{2}}^{ \pm}$follows the basic design strategy of Marquina's flux formula, developed in [13] for homogeneous conservation laws. At each cell-interface two states, $U^{L}$ and $U^{R}$, and the corresponding spectral decompositions of the Jacobian matrix $J(U)=\partial F / \partial U$, are computed at each side of the cell-interface by an appropriate nonlinear ENO interpolation process. Then, the numerical flux functions are obtained by applying the scalar algorithm to "sided" local characteristic variables and fluxes. Given $U^{L}=U_{i+\frac{1}{2}}^{L}$ and $U^{R}=U_{i+\frac{1}{2}}^{R}$, the left and right states at the $i+\frac{1}{2}$ cell-interface, the flux functions $G_{i+\frac{1}{2}}^{ \pm}$are computed as follows

$$
G_{i+\frac{1}{2}}^{ \pm}=\sum_{p=1}^{2}\left(\hat{G}_{i+\frac{1}{2}}^{ \pm}\right)^{p, L} R^{p}\left(U^{L}\right)+\left(\hat{G}_{i+\frac{1}{2}}^{ \pm}\right)^{p, R} R^{p}\left(U^{R}\right)
$$

where $L^{p}\left(U^{L}\right), R^{p}\left(U^{L}\right)\left(L^{p}\left(U^{R}\right), R^{p}\left(U^{R}\right), p=1,2\right.$, are the left and right eigenvectors of the Jacobian matrix $J(U)$. If $U_{i+\frac{1}{2}}^{L}=U_{i+\frac{1}{2}}^{R}=\bar{U}_{i+\frac{1}{2}}$ (e.g. $\bar{U}_{i+\frac{1}{2}}=\frac{U_{i}+U_{i+1}}{2}$ ), the resulting scheme, has the following simpler flux formula

$$
G_{i+\frac{1}{2}}^{ \pm}=\sum_{p=1}^{2}\left(\hat{G}_{i+\frac{1}{2}}^{ \pm}\right)^{p, L} R^{p}\left(\bar{U}_{i+\frac{1}{2}}\right)
$$

which is referred to as the $1 \mathrm{~J}$ scheme (for 1-Jacobian) in [6]. This scheme can be considered as a direct extension of the Shu-Osher schemes of [35] to a hyperbolic system of balance laws. 
As observed in [6], the 2-Jacobian (2J) scheme that results from considering the more complex flux formula in (21) has certain advantages, specially when dealing with wet/dry fronts, which involve two states (wet/dry) with very different properties, and for which a unique Jacobian at the interface is completely unphysical.

However, it is proven in [6] that if $U_{i+\frac{1}{2}}^{L}=U_{i+\frac{1}{2}}^{R}$ the scheme verifies the exact C-property, and if $U_{i+\frac{1}{2}}^{L} \neq U_{i+\frac{1}{2}}^{R}$ $(\mathbf{2 J})$, the scheme verifies only the approximate C-property, provided the order of accuracy is at least 2 . Hence, in [6] the authors propose the $\mathbf{1 J - 2 J}$ scheme, a numerical scheme that combines the two flux formulas to get the benefits of both alternatives. The 1J-2J scheme proposed in [6] is a high-cost numerical scheme, which can be combined with the multilevel strategy described above in order to obtain the high quality results of a HRSC numerical scheme on a fine mesh, at a lower computational cost.

\subsection{The TVDB scheme}

In [27], a completely different strategy is used in the design of a HRSC scheme. Second-order accuracy is achieved by considering a Lax-Wendroff type scheme for the scalar balance law (13), and using a flux-limiting procedure in order to curb the typical oscillations that appear when dealing with discontinuous profiles.

The following scheme is obtained in [27] from a Lax-Wendroff procedure applied to the pseudo-conservative form of the balance law (13),

$$
U_{i}^{n+1}=U_{i}^{n}-\frac{\triangle t}{\triangle x}\left(\mathcal{G}_{i+\frac{1}{2}}^{n}-\mathcal{G}_{i-\frac{1}{2}}^{n}\right)-\frac{\triangle t}{\triangle x} \mathcal{S}_{i}^{n}
$$

where

$$
\begin{aligned}
\mathcal{G}_{i+\frac{1}{2}}^{n} & =\frac{1}{2}\left(g_{i+1}^{n}+g_{i}^{n}-\alpha_{i+\frac{1}{2}}^{n}\left(g_{i+1}^{n}-g_{i}^{n}\right)\right), \\
\mathcal{S}_{i}^{n} & =\frac{1}{2}\left(\beta_{i+1 / 2}^{n}\left(g_{i+1}^{n}-g_{i}^{n}\right)+\beta_{i-\frac{1}{2}}^{n}\left(g_{i}^{n}-g_{i-1}^{n}\right)\right),
\end{aligned}
$$

and

$$
\left.\alpha_{i+1 / 2} \approx \frac{\triangle t}{\triangle x} f_{u}\right|_{i+1 / 2},\left.\quad \beta_{i+1 / 2} \approx \frac{\triangle t}{2} s_{u}\right|_{i+1 / 2} .
$$

This second order scheme is oscillatory when discontinuities are present in the solution, hence a limiting procedure is proposed in [27], order to curb the oscillations while maintaining the accuracy on smooth regions. The limited scheme is of the form shown in (23), but now $\mathcal{G}_{i+1 / 2}^{n}$ is defined as

$$
\mathcal{G}_{i+\frac{1}{2}}^{n}=\mathcal{G}_{i+\frac{1}{2}}^{L O}+\phi_{i+\frac{1}{2}}^{n}\left(\mathcal{G}_{i+\frac{1}{2}}^{H I}-\mathcal{G}_{i+\frac{1}{2}}^{L O}\right)
$$

where $\mathcal{G}_{i+\frac{1}{2}}^{H I}$ is given by (24) (it leads to a high order scheme), and $\mathcal{G}_{i+\frac{1}{2}}^{L O}$ is chosen so that its inclusion in (23) leads to a monotone (non-oscillatory) low order scheme. The following choice is considered in [27]

$$
\mathcal{G}_{i+\frac{1}{2}}^{L O}=\frac{1}{2}\left(g_{i+1}^{n}+g_{i}^{n}-\operatorname{sgn}\left(\alpha_{i+\frac{1}{2}}^{n}\right)\left(g_{i+1}^{n}-g_{i}^{n}\right)\right),
$$

where $\operatorname{sgn}(x)$ is the signum function. Finally, $\phi_{i+\frac{1}{2}}^{n}$ is an appropriate limiter which is defined as follows

$$
\phi_{i+\frac{1}{2}}^{n}=\max \left(0, \min \left(r_{i+\frac{1}{2}}^{n}, 1\right)\right)
$$


where $r_{i+\frac{1}{2}}$ measures the ratio of the upwind change to the local change, and is computed as

$$
r_{i+\frac{1}{2}}^{n}= \begin{cases}\frac{g_{i}^{n}-g_{i-1}^{n}}{g_{i+1}^{n}-g_{i}^{n}} & \operatorname{sgn}\left(\alpha_{i+\frac{1}{2}}^{n}\right)>0 \\ \frac{g_{i+2}^{n}-g_{i+1}^{n}}{g_{i+1}^{n}-g_{i}^{n}} & \operatorname{sgn}\left(\alpha_{i+\frac{1}{2}}^{n}\right)<0\end{cases}
$$

Carrying out the algebra, we obtain the following limited numerical flux function,

$$
\mathcal{G}_{i+\frac{1}{2}}^{n}=\mathcal{G}_{i+\frac{1}{2}}^{L O}+\phi_{i+\frac{1}{2}}^{n}\left(\operatorname{sgn}\left(\alpha_{i+\frac{1}{2}}^{n}\right)-\alpha_{i+\frac{1}{2}}^{n}\right)\left(g_{i+1}^{n}-g_{i}^{n}\right) .
$$

The numerical scheme that results from using the expression above is termed the TVDB scheme in [27]. The extension to systems is carried out using the characteristic-based approach mentioned above. Then, the scheme can be written as in (23), with $\mathcal{G}_{i+\frac{1}{2}}$ defined as

$$
\mathcal{G}_{i+1 / 2}^{n}=\sum_{p=1}^{2} \mathcal{G}_{i+1 / 2}^{n, p} R_{i+1 / 2}^{n, p} .
$$

Here $R_{i+1 / 2}^{n, p}$ are the right eigenvectors at the interface state, which we take as Roe mean of the states $U_{i}^{n}, U_{i+1}^{n}$ (other options are also possible). For the shallow water system the Roe mean requires the computation of the average values

$$
\hat{h}_{i+\frac{1}{2}}=\frac{1}{2}\left(h_{i}+h_{i+1}\right) \quad \text { and } \quad \hat{u}_{i+\frac{1}{2}}=\frac{\sqrt{h_{i}} u_{i}+\sqrt{h_{i+1}} u_{i+1}}{\sqrt{h_{i}}+\sqrt{h_{i+1}}} .
$$

The characteristic fluxes $\mathcal{G}_{i+1 / 2}^{n, p}$ are computed as in the scalar case (30), after pre-multiplying by the corresponding left eigenvectors, $L_{i+1 / 2}^{n, p}$.

A number of standard simulations are performed in [27] for scalar balance laws, obtaining always numerical approximations that seem to converge to the physically relevant solution. With respect to the well balancing property, it is observed in [27] that if the formal scheme, where the integrals of the source term are carried out in an exact fashion (this is possible in some cases), the scheme is exactly well balanced. If these integrals have to be numerically approximated, then the well balancing property depends on the integration rule used. For the shallow water system there is an intrinsic advantage in using the trapezoidal rule, since it can be shown that it gives the exact value of the integral of the source term on each computational cell for still-water steady states. We refer the reader to [27] and [6], for details on this matter, and simply remark here that, thank to this property, the schemes are exactly well balanced for still-water steady states.

\section{Numerical Experiments for Shallow Water Flows}

The two schemes described in the previous sections have the general form in (4), hence, our multilevel strategy can be applied almost as an external routine to the existing code, just as in the homogeneous case. Since the water height, $h$, contains all the non-smooth features of the flow, we use this variable in order to carry out the smoothness analysis that defines the flag vector. Our aim in this section is twofold, on one hand we shall present several numerical experiments that indicate that the multilevel strategy does not damage the well-balancing properties of the underlying scheme. On the other hand, we also evaluate the efficiency of the multilevel algorithm with respect to the reference simulation, estimated as the percentage of numerical divergences computed directly per time step, $\% f$, and also by $\theta_{i t e r}$, the CPU gain for a given iteration, and $\theta$, the gain for the global simulation, as in [8]. We include some tables that display these parameters in 2D 
simulations, and that indicate that the efficiency parameters are absolutely similar to those obtained in the homogeneous case $[8,9,32]$. In all experiments shown in this sections, the flag vector is computed as specified in (10) with $r=2$.

\subsection{D Numerical experiments}

A standard test to check the well balancing of a scheme in $1 \mathrm{D}$ steady smooth flow is given in e.g. [37]. It considers a channel of length $20 \mathrm{~m}$ with variable bathymetry, depicted in red in Figure 1, top-left and defined as

$$
z(x)=0.2 e^{-\frac{2}{5}(x-10)^{2}} .
$$

The quiescent state $h+z=2 \mathrm{~m}(q=0)$ is given as initial condition, and it is expected that a well-balanced scheme respects this equilibrium for all time.

In Figure 1, top-left, we show the free surface $h+z$ obtained after the application of the TVDB multilevel scheme after 50 seconds. Numerical results obtained with (a second order version of) the 1J-2J multilevel scheme are indistinguishable from those shown in Figure 1.

With the aim of evaluating the performance of a numerical scheme in the presence of complex and possibly non-smooth geometry, the following test was proposed in the workshop on dam-break wave simulation [18]. The initial data is the water at rest at a level of $12 \mathrm{~m}$. Numerical results obtained after a simulation of $200 \mathrm{~s}$ with the TVDB multilevel scheme are displayed in Figure 1, top-right. Numerical results obtained with the 1J-2J multilevel scheme are indistinguishable from those shown in Figure 1.

In Table 1 we display the $l_{1}$-error between the numerical solution obtained with the TVDB multilevel scheme and the true solution. In table 2 , we display the $l_{1}$-errors obtained after the application of the $1 \mathrm{~J}-2 \mathrm{~J}$ multilevel scheme.

\begin{tabular}{ccc}
\hline Grid size $\chi^{0}$ & Smooth $z(x)$ & Non-smooth $z(x)$ \\
\hline 128 & $4.2 \cdot 10^{-15}$ & $1.3 \cdot 10^{-16}$ \\
256 & $5.1 \cdot 10^{-14}$ & $1.4 \cdot 10^{-16}$ \\
512 & $2.3 \cdot 10^{-13}$ & $4.0 \cdot 10^{-15}$ \\
\hline
\end{tabular}

TABLE 1. Flow at rest: $l_{1}$-error obtained between the true solution and the numerical solution obtained with the multiscale TVDB scheme, with $L=5$, and $\varepsilon=10^{-3}$.

\begin{tabular}{ccc}
\hline Grid size $\chi^{0}$ & Smooth $z(x)$ & Non-smooth $z(x)$ \\
\hline 128 & $2.6 \cdot 10^{-15}$ & $2.6 \cdot 10^{-15}$ \\
256 & $5.2 \cdot 10^{-15}$ & $1.2 \cdot 10^{-14}$ \\
512 & $2.3 \cdot 10^{-14}$ & $2.5 \cdot 10^{-15}$ \\
\hline
\end{tabular}

TABLE 2. Flow at rest: $l_{1}$-error between the true solution and the numerical solution obtained using the multiscale $1 \mathrm{~J}-2 \mathrm{~J}$ scheme, with $L=5$, and $\varepsilon=10^{-3}$

The error, in all cases, is of the order of roundoff error in double precision computations, hence the tables indicate that the multilevel strategy preserves the exact C-property of the underlying HRSC technique, up to roundoff-type errors.

In Figure 1, middle (left and right), we show the water height. In Figure 1, bottom, the positions in which the flag vector has the value 1 are marked for each resolution level (marked as an integer in the y-axis of the 
figures). When the flow is smooth, very little effort is needed and, as we see in 1 bottom-left, only the lowest resolution levels are marked by the flag vector. On the other hand, when the bathymetry is non-smooth, the water height displays several non-smooth features which are correctly identified by the multiresolution data analysis performed within the multilevel strategy. This can be readily observed in Figure 1, bottom-right, where the positions identified by the flag vector in the various resolution levels are shown. We remind the reader that the costly numerical flux computations are carried out only at these positions, while the value of the 'extended' numerical divergence is being interpolated from lower resolution levels at all other locations of the grid $\chi^{0}$.

\subsection{D Numerical experiments}

As a validation of the exact C-property for 2D flows with smooth topography consider a smooth topography given by

$$
z(x, y)=0.8 e^{-50\left((x-0.5)^{2}+(y-0.5)^{2}\right)}
$$

with $(x, y) \in[0,1] \times[0,1]$ (see $[38])$. In table 3 , we show the measurements of the $l_{1}$-error between the exact quiescent steady state $\left(q_{1}=q_{2}=0\right)$ and the numerical results obtained with the $1 \mathrm{~J}-2 \mathrm{~J}$ multilevel scheme at $t=0.1 \mathrm{~s}$. We can see that the $l_{1}$-error is of the order of roundoff error. The exact C-property of the $1 \mathrm{~J}-2 \mathrm{~J}$ scheme is, thus, preserved. We also display the percentage of numerical divergences computed directly with the $1 \mathrm{~J}-2 \mathrm{~J}$ scheme per time step in the multilevel process.

\begin{tabular}{cccc}
\hline Grid size $\chi^{0}$ & $\% f$ & CPU gain $\theta$ & $l_{1}$-error \\
\hline $128 \times 128$ & 26.1102 & 2.8967 & $3.20 \cdot 10^{-16}$ \\
$256 \times 256$ & 20.5438 & 3.2427 & $3.49 \cdot 10^{-16}$ \\
$512 \times 512$ & 17.8262 & 3.9333 & $1.09 \cdot 10^{-15}$ \\
\hline
\end{tabular}

TABLE 3. Steady state with smooth topography. $l_{1}$-error between the computed solution with the 1J-2J multilevel scheme and the exact solution, $L=3, \epsilon=10^{-2}$. Percentage of computed 'extended' numerical divergences and CPU gain

Our next test is an extension to 2D of a 1D test due to LeVeque (see [38]). It involves a small perturbation of a steady state, and is used in the specialized literature in order to emphasize the importance of the well balancing property when computing small perturbations of steady flow. The domain is similar to that of the previous example, with the bottom topography given as

$$
z(x, y)=0.5 e^{-50\left((x-0.5)^{2}+(y-0.5)^{2}\right)}
$$

on $[0,1] \times[0,1]$ with $g=1$. The initial conditions are $q_{1}=q_{2}=0$ and

$$
h(x, y)= \begin{cases}1.01-z(x, y), & 0.1<x<0.2 \\ 1-z(x, y), & \text { otherwise }\end{cases}
$$

In Figure 2-(a), we display the level curves of the reference simulation, that is the numerical solution obtained without the multilevel algorithm. Figures 2-(b) and (c) show the level curves for multilevel simulations obtained with $\epsilon=10^{-3}$ and $\epsilon=10^{-4}$. The first threshold is too close to the value of the perturbation $\left(10^{-2}\right)$ and, as a consequence, the quality of the numerical solution is not sufficiently good. Small wiggles can be observed in the level curves of the computed solution. However, even at this large threshold, all the non-smooth features of the flow have been correctly identified and are marked by the flag vector, as observed in 2-(d). For $\epsilon=10^{-4}$, the level curves of the multilevel simulation are indistinguishable from those of the reference simulation. Figure 

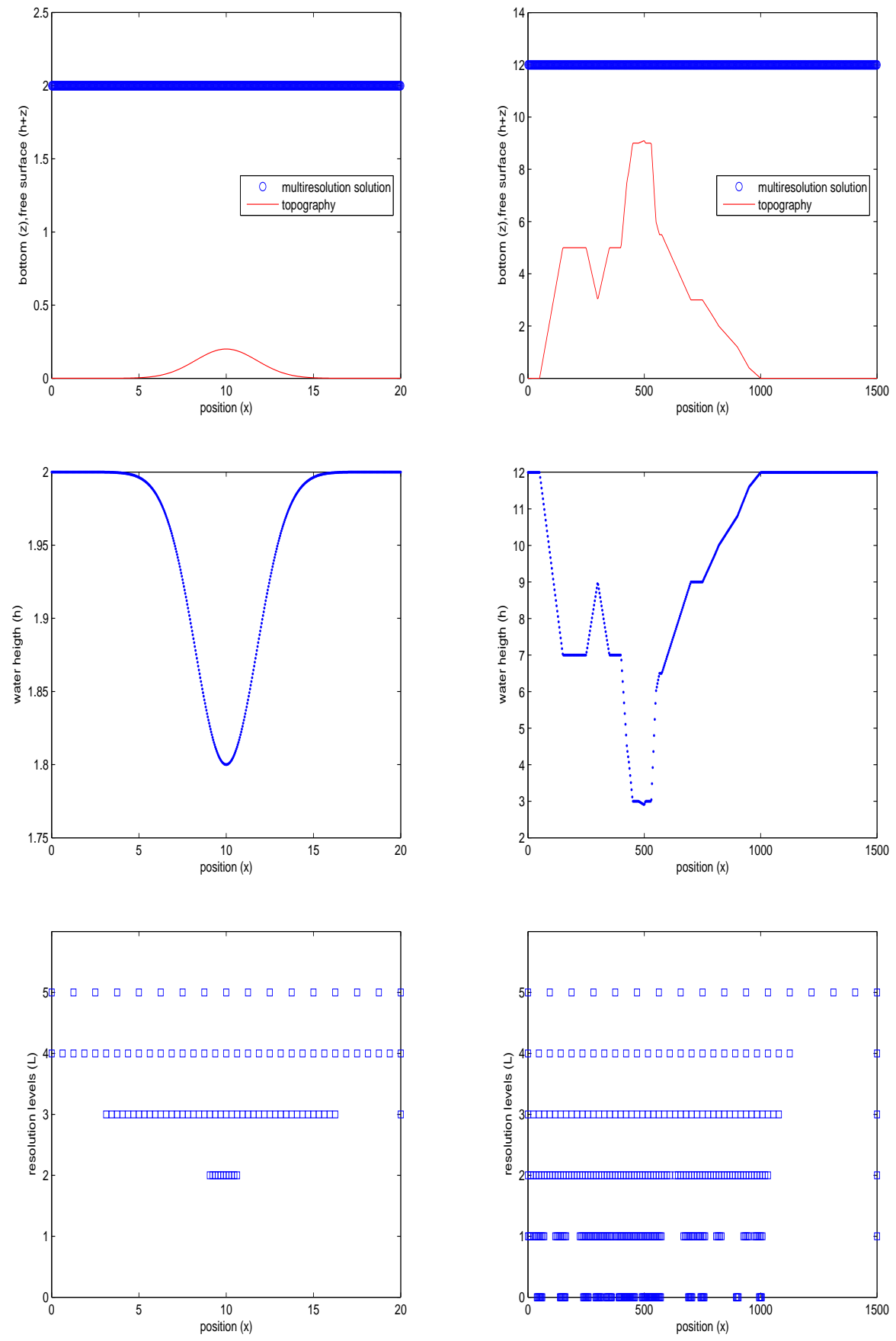

FIgURE 1. Flow at rest with the TVDB-multilevel scheme $(L=5)$. Left: Smooth topography, $t=50 \mathrm{~s}$. Right: Complex topography $t=200 \mathrm{~s}$. Top row: surface elevation, $h+z$. Middle row: water height, $h$. Bottom row: location of points where the flag vector takes the value 1 , at resolution levels $l=0,1, \ldots L . \varepsilon=10^{-3}$. 


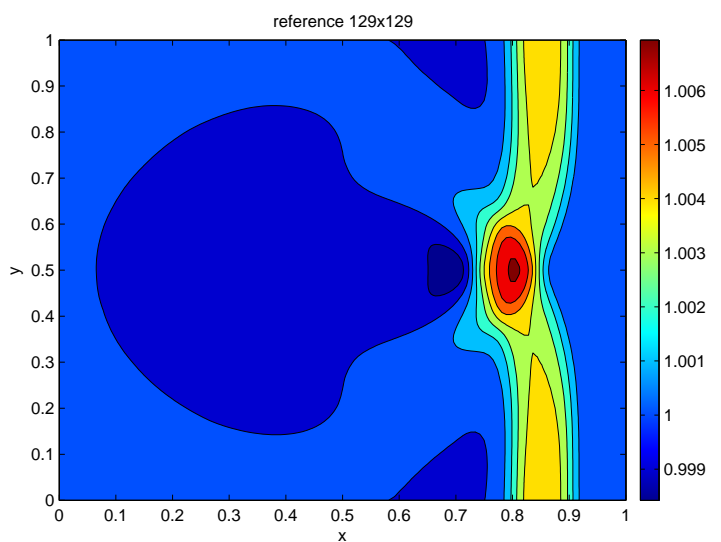

(a)

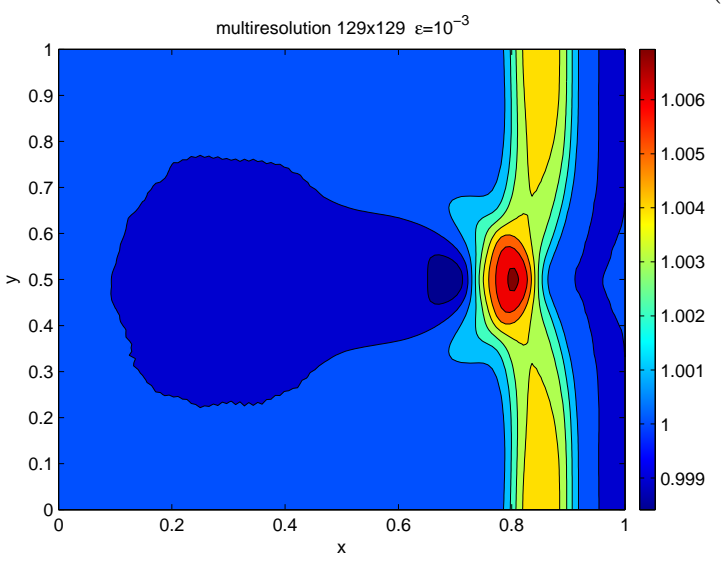

(b)

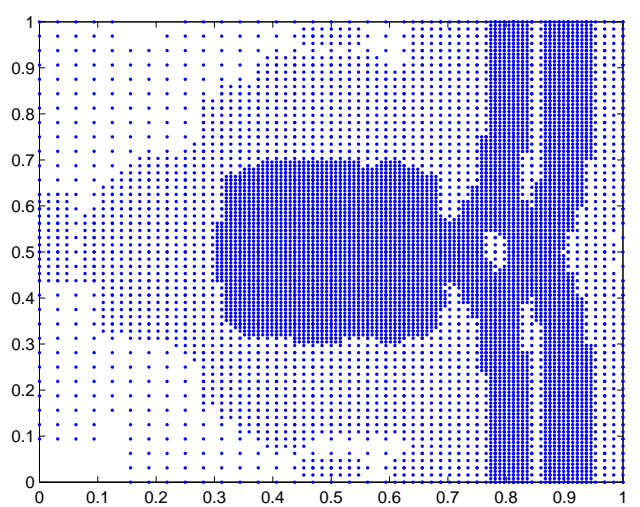

(d)

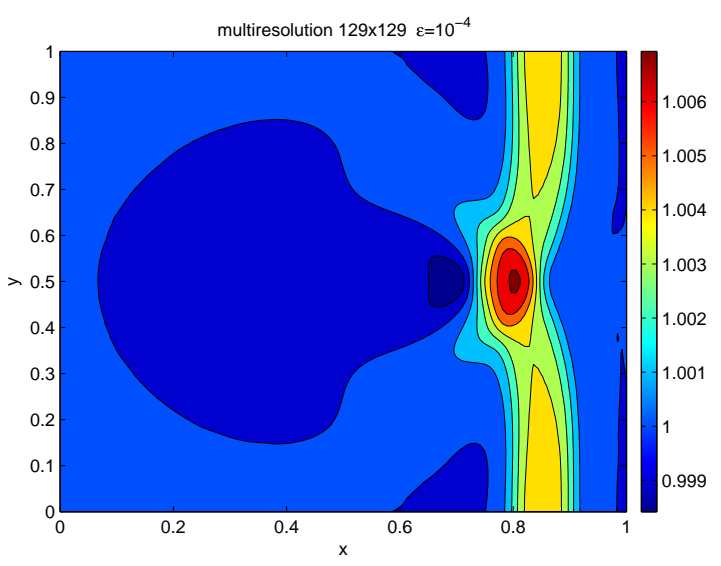

(c)

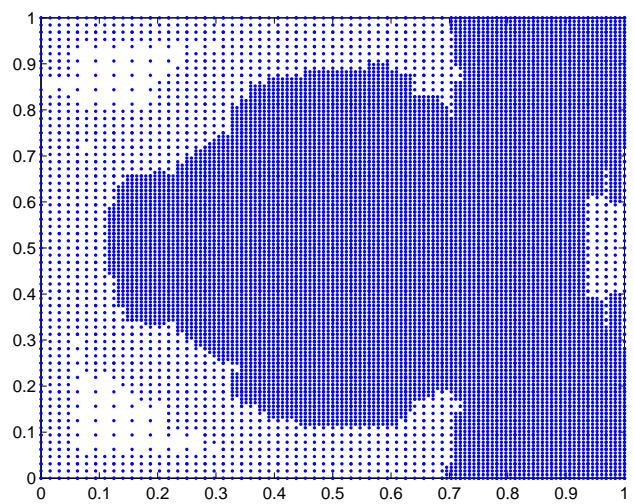

(e)

Figure 2. LeVeque 2D test at time $t=0.7$. Level curves of (a) reference solution. (b) multilevel simulation with $\varepsilon=10^{-3}$. (c) multilevel simulation with $\varepsilon=10^{-4}$. Points of $\chi^{0}$ where the flag vector has the value 1 , (d) for $\varepsilon=10^{-3}$; (e) For $\varepsilon=10^{-4}$. 
2-(e) shows the locations, on the finest grid $\chi^{0}$, where the flag vector has the value 1 . Only three levels have been used, for visual clarity.

Finally, we consider a test problem from [7], where the true solution is not known. The domain is the square $[0,2] \times[0,2]$ and the topography is given by the function:

$$
z(x, y)= \begin{cases}\frac{1}{8}(\cos (2 \pi(x-0.5))+1)(\cos (2 \pi y)+1), & (x-1.5)^{2}+(y-1)^{2} \leq(0.5)^{2} \\ 0, & \text { otherwise }\end{cases}
$$

The initial conditions are $q_{1}=q_{2}=0$ and

$$
h(x, y)= \begin{cases}1.1-z(x, y), & (x-1.25)^{2}+(y-1)^{2} \leq(0.1)^{2} \\ 0.6-z(x, y), & \text { otherwise }\end{cases}
$$

In Figure 3 we show a comparison between the solutions obtained with and without the multilevel algorithm at different times $(t=0.1,0.2)$, to allow for a direct comparison with the results in [7]. In Table 4 we display the global gain for each simulation and the maximum and the minimum values for $\% f$ in the simulation. As in the homogeneous case, we can observe that the finer the grid the smaller the percentage of direct evaluations of the extended divergence, and the higher the gain, maintaining a quality in the computed solution which is directly related to the value of the threshold coefficient in (10).

\begin{tabular}{cccccc}
\hline Grid size $\mathcal{G}^{0}$ & $\% f_{\min }$ & - & $\% f_{\max }$ & cpu gain $\theta$ & $l^{1}-$ error \\
\hline $128 \times 128$ & 16.78 & - & 29.99 & 2.89 & $1.70 \cdot 10^{-5}$ \\
$256 \times 256$ & 9.19 & - & 19.58 & 3.83 & $1.24 \cdot 10^{-5}$ \\
$512 \times 512$ & 5.33 & - & 10.87 & 5.04 & $2.41 \cdot 10^{-5}$ \\
\hline
\end{tabular}

TABle 4. Circular Dam break test at time $t=0.1$. Percentage of extended divergences computed with the scheme, CPU gain and $l_{1}$-error

\section{The Penalized COMPressible Navier-Stokes Equations}

When dealing with realistic flow simulations, the development of efficient numerical schemes can be particularly tricky due to the presence of complex geometries. Many different techniques have been investigated, from coordinate transformations and body fitted structured and unstructured grids to fictitious domain approaches. A different set of techniques stems from the penalization method introduced by E. Arquis and J.P. Caltagirone [2] and analyzed for the incompressible Navier Stokes equations by Angot et al. in [1]. The obstacles are described by a mask function and the momentum equations in the Navier-Stokes system are modified by adding a supplementary term with the idea of forcing the velocity to satisfy the no-slip conditions on the body of the obstacle. Since the new penalized system is solved in an obstacle-free domain, fast and effective methods for Cartesian grids can be used. Different numerical simulations of viscous flows using adaptive wavelet methods $[29,34,36]$, pseudospectral methods $[23,28,31]$, finite difference/volume methods $[1,30]$ or vortex method [11] have put in evidence the efficiency of this technique for incompressible flow simulations.

In [5], we proposed an efficient penalization technique for the compressible Navier-Stokes equations. Numerical simulations of interactions between obstacles and high Mach number flows have been performed, showing the robustness of this method for shocked flows, from the point of view of consistency with physical principles. In what follows, we recall the essential ingredients of the penalization technique developed in [5] and focus on the benefits of the coupling with the multilevel technique described in section 1. 

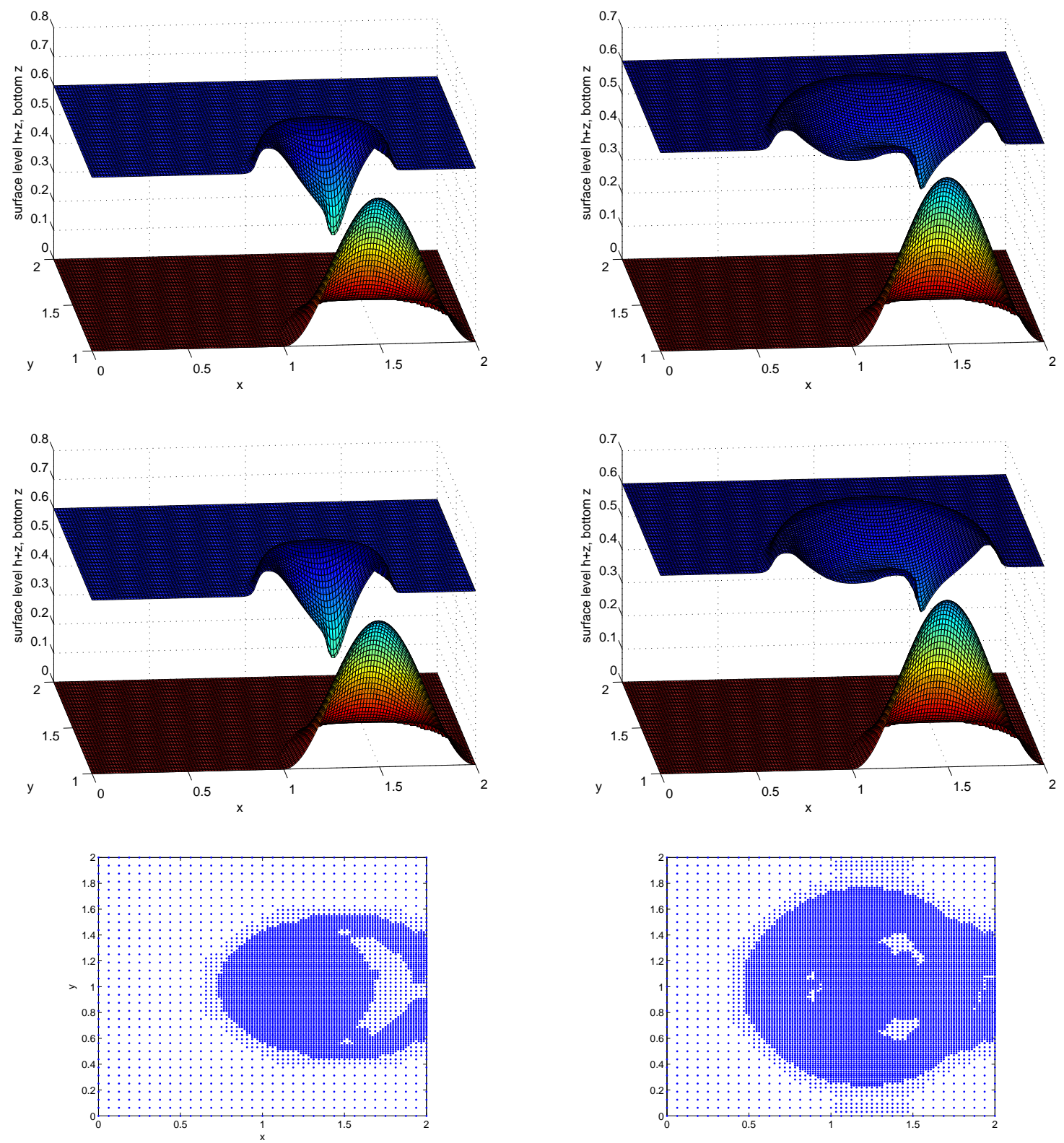

Figure 3. Circular Dam Break test at time $t=0.1$ (left) and $t=0.2$ (right). From top to bottom: free surface and bottom topography (slice of the channel at $y=1$ ) for the reference solution, free surface and bottom topography of the multilevel simulation with $\varepsilon=10^{-3}$, points of $\chi^{0}$ where the numerical divergence is computed for $\varepsilon=10^{-3}$.

We consider a computational domain $\Omega$, made up of a fluid domain $\Omega_{f}$ and a solid domain $\Omega_{s}$, i.e $\Omega=\Omega_{f} \cup \Omega_{s}$. The classical compressible Navier Stokes system can be written in the form (1) with $\vec{U}=(\rho, \rho u, \rho v, E)^{T}$ being the dimensionless vector of unknowns and $\mathcal{B}=\frac{1}{R e} \vec{\nabla} \mathcal{F}_{V}(\vec{U})$, where $R e$ is the Reynolds number and $\mathcal{F}_{V}(\vec{U})$ represents the viscous fluxes in the compressible Navier-Stokes equations (see e.g. [22]). In general, (1) is solved 
only on the fluid domain, $\Omega_{f}$, after imposing appropriate conditions on the boundary of the solid, $\Gamma_{s}$. Assuming that we impose no-slip boundary conditions on $\Gamma_{s}$ for the velocity vector, and a Dirichlet condition on the temperature of $\Gamma_{s}$, the penalization technique considers a new system of the form (1), where $\mathcal{B}$ is replaced by

$$
\mathcal{B}_{\eta}=\mathcal{B}-\frac{\mathbf{1}_{\Omega_{s}}}{\eta}\left(\begin{array}{c}
0 \\
\rho u \\
\rho v \\
E-E_{\Omega_{s}}
\end{array}\right) .
$$

Here $\mathbf{1}_{\Omega_{s}}$ is the characteristic function of the solid domain and $\eta$ denotes the penalization parameter, $\eta<<1$. The penalization term includes $E_{\Omega_{s}}$, the 'energy' of the solid body $\Omega_{s}$, whose value is given in terms of the fixed wall temperature $T_{\Omega_{s}}$ and the perfect gas law by

$$
E_{\Omega_{s}}=\rho c_{\mathrm{v}} T_{\Omega_{s}}
$$

The resulting penalized system is now solved in the entire domain $\Omega$ with a given initial condition $\vec{U}_{0}(X)$. Using a formal asymptotic expansion of the variables in the parameter $\eta$, we observe in [5] that the conditions

$$
u=v=0 \quad \text { and } \quad T=T_{\Omega_{s}}
$$

should be recovered inside the solid $\Omega_{s}$ by this technique. This fact is confirmed by the numerical experiments shown in [5], where it is observed that, inside the obstacle, the velocity converges to zero with respect to the penalization parameter at a rate proportional to $\eta$. This same rate is reported in many papers where a similar penalization technique is employed for incompressible flows. The formal asympotic analysis study perfomed in [5] reveals that the classical Navier-Stokes equations are recovered in the fluid domain.

\subsection{Multilevel-HRSC schemes for the penalized system}

In order to obtain numerical simulations involving supersonic flows, the convective terms must be discretized with a HRSC scheme. In [5], we use a combination of Marquina's solver [13,14] with a third order non-linear reconstruction procedure [26] to evaluate the inviscid fluxes (3) in the penalized system. The discrete time evolution is obtained with a third order TVD Runge-Kutta method [35]. The viscous fluxes are handled explicitly (with centered finite difference formula) since the simulations involve flow with very large Reynolds number $\left(R e \sim 10^{4}\right)$. The penalization term, however, is discretized in an implicit fashion, in order to keep the numerical stability condition independent of $\eta$, which is chosen to be very small. However, the specific form of this term leads to a diagonal linear system to be solved at each time step, hence no extra work is involved in practice in comparison to a fully explicit discretization.

In [5], this algorithm has been applied to simulate the interactions of supersonic flows with obstacles and the results have been analyzed in regards to theoretical well known properties of these flows. The reported simulations show for example a very good agreement for shape and angles of bow shocks or for drag coefficients, once the numerical mesh is fine enough. This is not surprising since the obstacles are only represented by the mask function $\mathbf{1}_{\Omega_{s}}$ whose discretization on a Cartesian grid leads to a piecewise constant description of the boundary $\Gamma_{s}$. A geometrical error of size $o(h)$, where $h$ is the mesh size, is therefore always introduced and a fine mesh is required around the boundaries in order to decrease this error. This feature makes the coupling between the basic HRSC algorithm and the multilevel techique advocated in this paper all the more relevant.

From a purely computational point of view, an apparent drawback of the penalization method comes from the fact that the penalized system is also solved inside of the obstacles, where the quantities computed are totally non physical. Depending on the relative sizes of $\Omega_{s}$ and $\Omega_{f}$, the CPU time could indeed increase significantly, 
and the advantages of this technique can be lost in comparison to body-fitted classical methods. However, the combination with the multilevel algorithm presented in section 1 can considerably reduce these adverse effects, in particular since it has been observed in a large battery of numerical tests (see also figure 4) that the penalized solution remains nearly constant inside an obstacle, hence very little resolution is needed in its computation.

For the type of robust HRSC scheme used in our simulations, the computation of the numerical inviscid fluxes with the HRSC scheme represents about $90 \%$ of the global CPU time. Therefore, it is natural to evaluate these fluxes with the multiresolution method of section 1 in order to try to reduce the computational effort. Here, the computation of $\mathcal{B}_{\eta}$ is straightforward in comparison to the shallow water system and is made directly on the finest computational grid.

Figure 4 presents the results obtained for a right-going shock wave interacting with a triangular shaped obstacle. The pressure after the interaction is represented on 4(a). Thanks to the multiresolution process, the robust solver is only used around the large variations areas of the flow, as observed on 4(b). In the obstacle the velocity and temperature are enforced to constant values by the penalization technique, but all the other variables are also nearly constant except inside of a thin boundary layer located at the frontier as shown on figure 4(c)-(d) for the pressure. Therefore, most of the numerical convective fluxes are interpolated instead of computed by the solver. In the present case, only $54 \%$ of the numerical divergence is computed with the HRSC scheme in the obstacle. This percentage for the full computational domain grows during the simulation from 4 to 18 , leading to a reduction of $\mathrm{CPU}$ time by a factor $\theta=5.1$.

The penalization method for the compressible Navier Stokes system combined with the multiresolution scheme allows to handle immediately complex geometries at reasonable computational time, only changing the mask function $\mathbf{1}_{\Omega_{s}}$. As an example, the figure 5 represents the modification of a converging circular shock wave due to the presence of cylinders. This configuration is used experimentally in $[15,16]$ in order to modify the shape of the shock before focussing and its influence on the amount of emitted light. Converging polygonal shaped shock waves are easily created in this way. Our numerical technique is able to represent the qualitative behavior of the flow and to reproduce triangular or square shaped shock waves as observed experimentally and numerically in $[15,16]$. The 3 - and 4-cylinder simulations have been run on a $512^{2}$ grid and the multiresolution algorithm leads respectively to $\theta=3.8$ and $\theta=3$ reduction factor. The 12 -cylinder experiment use a $1024^{2}$-mesh and the CPU time reduction is only of a factor 2.1 due to the very complex structure of the flow.

\section{Conclusions}

Cost-effective multilevel schemes, based on Harten's multiresolution framework, provide a versatile tool that can be easily combined with an existing HRSC code, in order to reduce the amount of computational time employed in fine-mesh numerical simulations. The technique is especially valuable in $2 \mathrm{D}$, where very often the computational expense associated to some HRSC schemes can prevent the use of personal computers in the testing process.

The multilevel strategy based on Harten's interpolatory framework has proven to be a useful asset in the development of new numerical techniques for problems that need a specialized numerical treatment around shocks and other singularities in the flow.

The numerical treatment of source terms can be a delicate issue in some instances, as exemplified by the problems considered in this paper. We have shown that the multilevel technique in [8], described originally for homogeneous conservation laws, can be easily adapted to some convection-dominated problems with source terms, providing high resolution simulations on fine meshes at lower computational cost.

For shallow water flows, we show that the well-balancing properties of the underlying numerical scheme are respected by the multilevel technique. For compressible flow with obstacles, the use of the penalization technique requires an implicit treatment of the stiff penalization term, which is handled easily within the multilevel strategy. In this latter case, we also remark that the penalization method requires the computation 


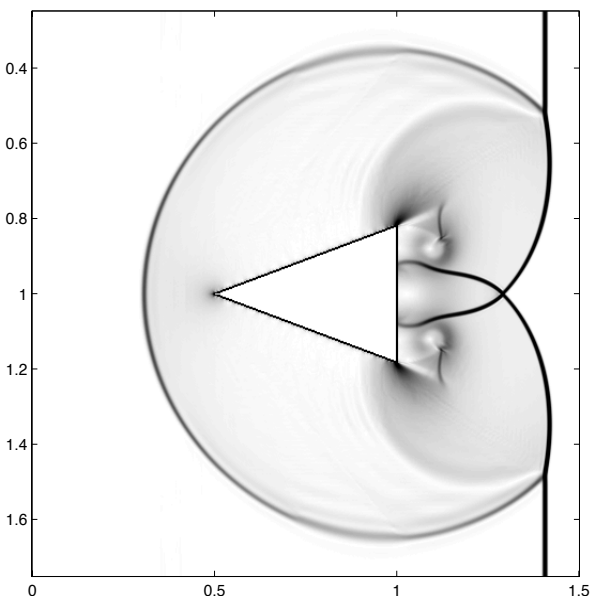

(a)

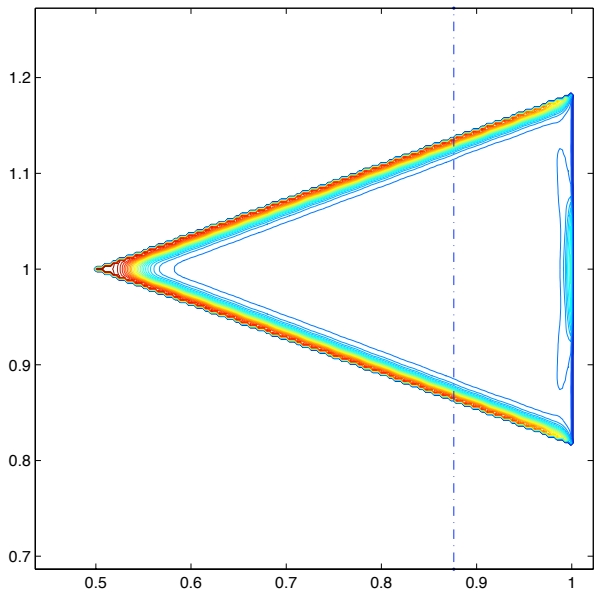

(c)

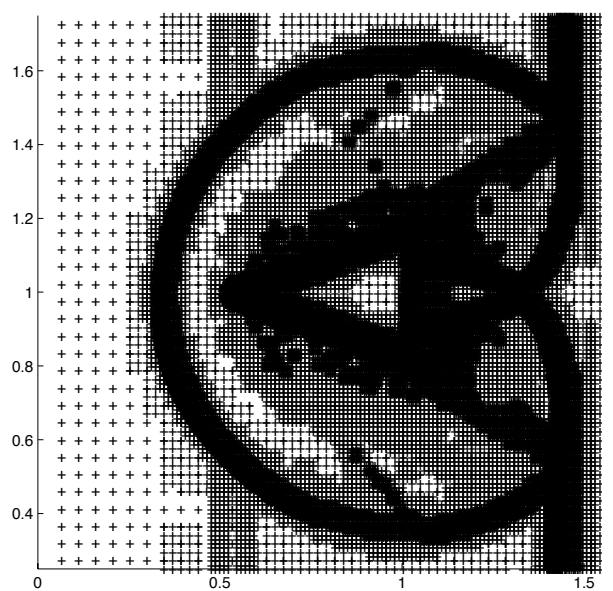

(b)

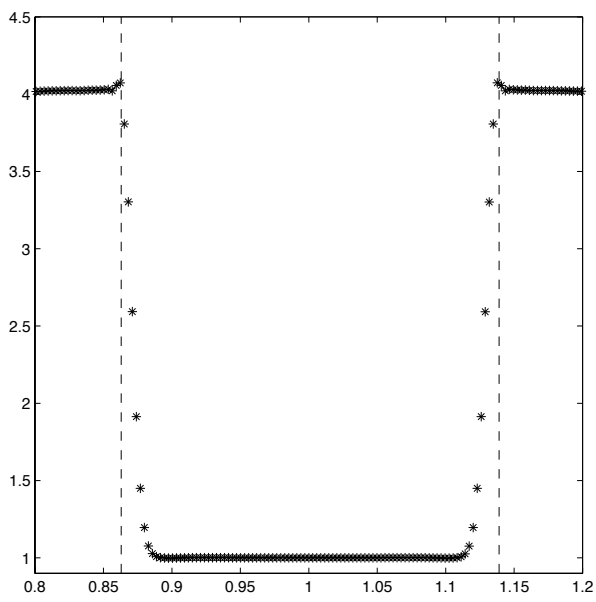

(d)

FIGURE 4.

(a): Schlieren representation of the pressure after a Mach 1.7 shock-triangle interaction. (b): Representation of the points of $\chi^{0}$ where the HRSC scheme has been used for evaluation of the numerical divergence. (c):

Contour lines of the pressure inside of the obstacle. (d): Computed pressure along the dotted line of figure (c), vertical dashed line represents the boundaries of the triangle. Simulation obtained with $\chi^{0}=512^{2}$ points, $L=5$ levels of multiresolution and the thresholding parameter $\epsilon=5.10^{-4}$.

of the numerical solution inside the obstacle, which is non-physical but turns out to be nearly constant inside the obstacle. This fact is exploited by the multilevel technique in an automatic manner, therefore very little resolution, and hence very little computational effort, is employed inside the obstacles.

\section{REFERENCES}

[1] Ph. Angot, C.-H. Bruneau, and P. Fabrie. A penalization method to take into account obstacles in incompressible flows. Numer. Math., 81(4):497-520, 1999.

[2] E. Arquis and J.P. Caltagirone. Sur les conditions hydrodynamiques au voisinage d'une interface milieu fluide-milieux poreux: application la convection naturelle. C.R. Acad. Sci. Paris II, 299:1-4, 1984. 


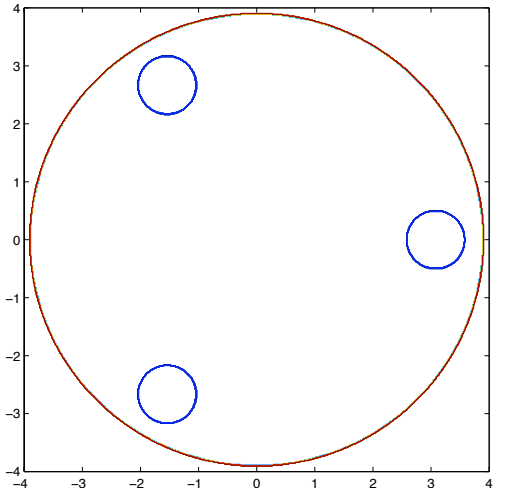

(a)

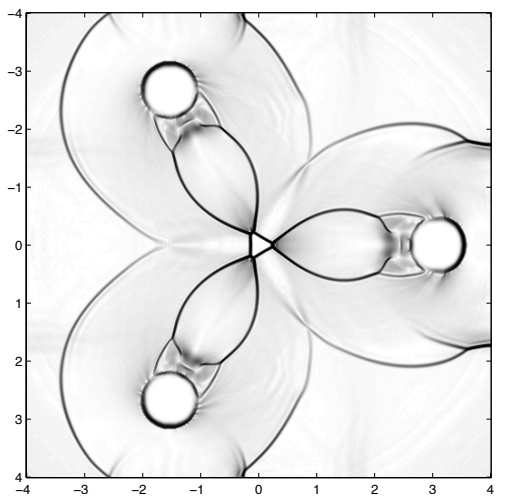

(c)

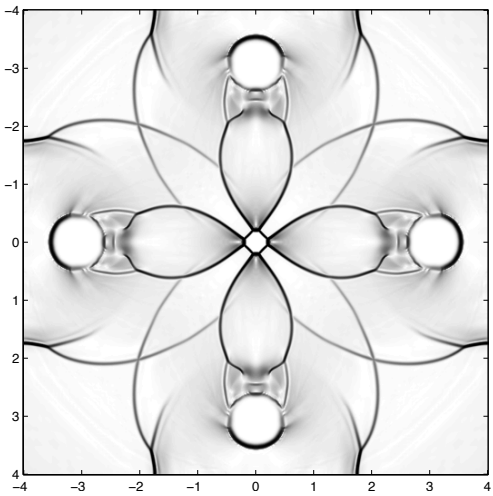

(e)

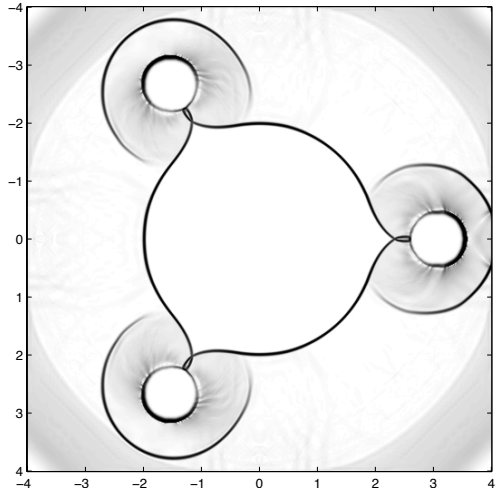

(b)

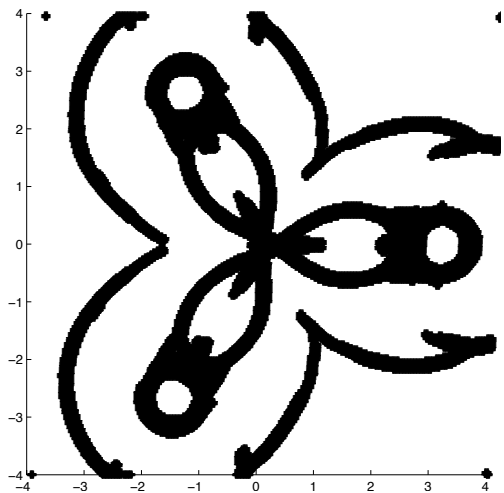

(d)

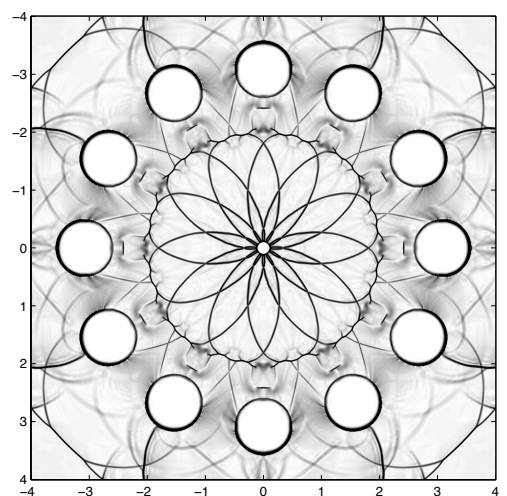

(f)

FIgURE 5. Converging shock wave-cylinders interaction. (a): initial condition for 3 obstacles. (b)-(c) Schlieren representation of the pressure at different times. (d): Points of $\chi^{0} / \chi^{1}$ where the multilevel divergence is computed by the solver. (e)-(f): same simulations with 4 and 12 cylinders.

[3] A. Bermúdez and M. E. Vázquez. Upwind methods for hyperbolic conservation laws with source terms. Computers 85 Fluids, 23:1049-1071, 1994.

[4] B. L. Bihari and A. Harten. Multiresolution schemes for the numerical solution of 2-D conservation laws. I. SIAM J. Sci. Comput., 18(2):315-354, 1997. 
[5] O. Boiron, G. Chiavassa, and R. Donat. A high-resolution penalization method for large mach number flows in the presence of obstacles. Computers and Fluids, 38(3):703-714, 2009.

[6] V. Caselles, R. Donat, and G. Haro. Flux gradient and source term balancing for certain high resolution shock capturing schemes. Submitted.

[7] M. J. Castro, E. D. Fernández-Nieto, A. M. Ferreiro, J. A. García-Rodríguez, and C. Parés. High order extensions of Roe schemes for two-dimensional nonconservative hyperbolic systems. J. Sci. Comput., 39(1):67-114, 2009.

[8] G. Chiavassa and R. Donat. Point value multiscale algorithms for 2D compressible flows. SIAM J. Sci. Comput., 23(3):805-823, 2001.

[9] G. Chiavassa, R. Donat, and A. Marquina. Fine-mesh numerical simulations for 2d Riemann problems with a multilevel scheme. International Series of Numerical Mathematics (Birkhäuser), 140, 2001.

[10] A. Cohen, S. M. Kaber, S. Müller, and M. Postel. Fully adaptive multiresolution finite volume schemes for conservation laws. Math. Comp., 72(241):183-225, 2003.

[11] M. Coquerelle and G.-H. Cottet. A vortex level set method for the two-way coupling of an incompressible fluid with colliding rigid bodies. J. Comput. Phys., 227(21):9121-9137, 2008.

[12] M.O. Domingues, O. Roussel, and K. Schneider. Global time step control in adaptive multiresolution methods for pdes. submitted to Int. J. Numer. Meth. Engin., (2), 2007.

[13] R. Donat and A. Marquina. Capturing shock reflections: An improved flux formula. J. Comput. Phys., 125:42-58, 1996.

[14] R. Donat and P. Mulet. The two-Jacobian scheme for systems of conservation laws. In Analysis and Simulation of Fluid Dynamics, pages 89-108. Birkhäuser, 2007.

[15] V. Eliasson, N. Apazidis, and N. Tillmark. Controlling the form of strong converging shocks by means of disturbances. Shock waves, 17(1-2):29-42, 2007.

[16] V. Eliasson, W.D. Henshaw, and D. Appelö. On cylindrically converging shock waves shaped by obstacles. Physica D: Nonlinear Phenomena, 237(14-17):2203-2209, 2008.

[17] Ll. Gascón and J. M. Corberán. Construction of second-order TVD schemes for nonhomogeneous hyperbolic conservation laws. J. Comput. Phys., 172(1):261-297, 2001.

[18] N. Goutal and F. Maurel, editors. Proceedings of the 2nd Workshop on Dam Break Wave Simulation. EDF-DER Report HE-43/97/016/B, 42,45, 1997.

[19] J. M. Greenberg and A. Y. Leroux. A well-balanced scheme for the numerical processing of source terms in hyperbolic equations. SIAM J. Numer. Anal., 33(1):1-16, 1996.

[20] J. M. Greenberg, A. Y. Leroux, R. Baraille, and A. Noussair. Analysis and approximation of conservation laws with source terms. SIAM J. Numer. Anal., 34(5):1980-2007, 1997.

[21] A. Harten. Multiresolution algorithms for the numerical solution of hyperbolic conservation laws. Comm. Pure Appl. Math., 48(12):1305-1342, 1995.

[22] C. Hirsch. Numerical Computation of Internal and External Flows. Volume 1: Fundamentals of Numerical Discretization. Wiley Series in Numerical Methods in Engineering.

[23] G. H. Keetels, U. D'Ortona, W. Kramer, H. J. H. Clercx, K. Schneider, and G. J. F. van Heijst. Fourier spectral and wavelet solvers for the incompressible navier-stokes equations with volume-penalization: Convergence of a dipole-wall collision. $J$. Comput. Phys., 227(2):919-945, 2007.

[24] P. Lamby, S. Müller, and Y. Stiriba. Solution of shallow water equations using fully adaptive multiscale schemes. Internat. J. Numer. Methods Fluids, 49(4):417-437, 2005.

[25] R. J. LeVeque. Balancing source terms and flux gradients in high-resolution Godunov methods: the quasi-steady wavepropagation algorithm. J. Comput. Phys., 146(1):346-365, 1998.

[26] A. Marquina. Local piecewise hyperbolic reconstruction of numerical fluxes for nonlinear scalar conservation laws. SIAM $J$. Sci. Comput., 15(4):892-915, 1994.

[27] A. Martínez Gavara. High Resolution Schemes for Hyperbolic Conservation Laws with Source Terms. PhD thesis, Universitat de València, 2008.

[28] M. Minguez, R. Pasquetti, and E. Serre. High-order large-eddy simulations of flow over the ahmed body car model. Phys. Fluids, 20(9):095101, 2008.

[29] O.V. Vasilyev N.K.R Kevlahan. An Adaptive Wavelet collocation Method for Fluid-Structure Interaction at High Reynolds Number. SIAM J. Sci. Compt., 26(6):1894-1915, 2005.

[30] A. Paccou, G. Chiavassa, J. Liandrat, and K. Schneider. A penalization method applied to the wave equation. Comptes Rendus Mécanique, 333/1, 2005.

[31] R. Pasquetti, R. Bwemba, and L. Cousin. A pseudo-penalization method for hight Reynolds number unsteady flows. Appl. Numer. Math., 58(7):946-954, 2007.

[32] A. Rault, G. Chiavassa, and R. Donat. Shock vortex interaction at high mach numbers. J. Sci. Comput., 19:347-371, 2003.

[33] O. Roussel, K. Schneider, A. Tsigulin, and H. Bockhorn. A conservative fully adaptive multiresolution algorithm for parabolic PDEs. J. Comput. Phys., 188(2):493-523, 2003.

[34] K. Schneider and M. Farge. Adaptive wavelet simulation of a flow around an impulsively started cylinder using penalisation. Applied and Computational Harmonic Analysis, 12(3):374-380, May 2002. 
[35] C.W. Shu and S. Osher. Efficient implementation of essentially nonoscillatory shock-capturing schemes. II. J. Comput. Phys., 83(1):32-78, 1989.

[36] O.V. Vasilyev and N. Kevlahan. Hybrid wavelet collocation-brinkman penalization method for complex geometry flows. Int. J. Num. Meth. Fluids, 40:531-538, 2002.

[37] S. Vukovic and L. Sopta. ENO and WENO schemes with the exact conservation property for one-dimensional shallow water equations. J. Comput. Phys., 179(2):593-621, 2002.

[38] Y. Xing and C.W. Shu. High order finite difference WENO schemes with the exact conservation property for the shallow water equations. J. Comput. Phys., 208(1):206-227, 2005. 